\title{
A review on role of trichomes in plant physiology and genetic mechanism involved in trichome regulation in cotton
}

\author{
Muhammad Shahzad ${ }^{1}$, Zulqurnain Khan ${ }^{1}$, Syed Ismat Hussain ${ }^{2}$, Adeela \\ Rasheed $^{1}$, Maria Basheer ${ }^{3}$, Khadim Hussain ${ }^{4}$, Muhammad Tauseef ${ }^{5}$, \\ Javed Iqbal ${ }^{6}$ and Wajad Nazeer ${ }^{6 *}$ \\ 1. MNS-University of Agriculture Multan-Pakistan \\ 2. Pest Warning \& Quality Control of Pesticides, Multan-Pakistan \\ 3. Institute of Molecular Biology and Biotechnology, Bahauddin Zakarya University Multan-Pakistan \\ 4. Central Cotton Research Institute, Multan-Pakistan \\ 5. Agronomic Research Institute, Ayub Agricultural Research Institute, Faisalabad-Pakistan \\ 6. Cotton Research Institute, Multan-Pakistan \\ *Corresponding author's email: wajidpbg@yahoo.com \\ Citation \\ Muhammad Shahzad, Zulqurnain Khan, Syed Ismat Hussain, Adeela Rasheed, Maria Basheer, Khadim Hussain, \\ Muhammad Taouseef, Javed Iqbal and Wajad Nazeer. Malaria burden. Pure and Applied Biology. Vol. 10, Issue \\ 2, pp458-464. http://dx.doi.org/10.19045/bspab.2021.100049
}

\begin{tabular}{llll}
\hline \hline Received: 09/06/2020 & Revised: 15/09/2020 & Accepted: 07/10/2020 & Online First: 24/10/2020 \\
\hline \hline
\end{tabular}

\section{Abstract}

Natural hair like structures, produced on aerial surfaces of plants that involves in plant defence, are recognised as trichomes. These trichomes play their role in natural plant defence. These trichomes may be glandular or non-glandular, and secrete exudates, which trap insects and slowdown their movement and act as a physical barrier either against insect attack killing the insects directly or through retarding the insect growth and their population. These trichomes play an important role against both biotic and abiotic stresses. They also play a vital role in developing resistance against sucking insects like whitefly. Genes involved in trichomes development are TTG1, GL1, GL2, GL3 and MYB gene in Arabidopsis and cotton. There are some negative regulators in trichomes development like CPC, TCL1 and TCL2 reported in Arabidopsis. Phytohormones like jasmonic acid, salisyllic acid are also involved in trichomes development. Trichome density is also equally important and can be measured by scaling method and we can categorize leaves based on trichome density. We had reviewed the trichomes, its classification, morphology, different genes responsible for trichome regulation and role of different phytohormones in regulation of trichomes that can be helpful for classification of available germplasm of cotton and other crops.

Keywords: Negative regulators; Physical barrier; Phytohormones; Transparent testa glabra; Trichomes

\section{Introduction}

Cotton is an important cash crop in Pakistan. It serves as a jugular vein for economy of the country. It plays crucial role in GDP of the country. Cotton accounts for $1 \%$ of total agriculture GDP. Pakistan is $3^{\text {rd }}$ largest exporter and yarn producer of cotton, $2^{\text {nd }}$ in yarn exports and $4^{\text {th }}$ largest consumer of cotton in the world [1].

Various biotic and abiotic factors are responsible for reduction in cotton yield in recent years. Biotic factors like insect attack cause major reduction in cotton production in both quality and quantity of the produce. 
Like cotton leaf curl disease, for whitefly serves as vector, causing major economic losses for cotton production. Drought and salinity also play important role in cotton production. These stresses are also responsible for decrease in cotton production.

Trichomes are hair like structures, which are produced on plant surface (Fig. $1 \& 2$ ). Among plant parts, leaf trichomes are the most important. Trichomes may be glandular or non-glandular. These appendages present on plant acts as an important tool against insect attack. Glandular trichomes secrete exudates, which prevent insects attack by entrapping them [2]. The outstanding property of glandular trichomes is their unique capacity to synthesize, store and sometimes secrete a wide range of metabolites including polysaccharides, organic acids, proteins, alkaloids, and polyphenols. These compounds provide a distinctive smell to plants acts as natural barrier to protect plants against herbivorous insects and pathogens and have an important commercial value as drugs, fragrances, food additives [3].

Different types of trichomes protect the plants in quite different ways. Some kinds of trichomes may deliver a plant with defence against herbivory; while, some hairs may shield against frost damage. Some types of hairs i.e. trichomes provide protection against water loss by break up the flow of air across a plant's surface [4]. Non-glandular trichomes are involves for reduction in heat load, increase tolerance to freezing or drought, facilitate water absorption and protect from UV light and insect herbivores [5]. Glandular trichomes provide chemicals against herbivores and pathogens for trapping insects.

Trichomes also have developmental roles in regulating flower structure. Silencing a master regulator of petal trichomes, GhMYB-MIXTA-Like10

(GhMYBML10), by RNA interference (RNAi) suppresses petal trichome growth and results in flower buds formation into abnormal curved shape that exposes to developing anthers and stigmas to desiccation damage. The cotton 'Velcro' represents a novel function of trichomes: binding different floral parts together to shape their growth and help successful reproduction [6].

Trichomes also serves as physical barriers against insect attack. Trichomes density and length changes with different conditions like in case of herbivore attack, trichomes become denser and its length also increase (Fig. 3).

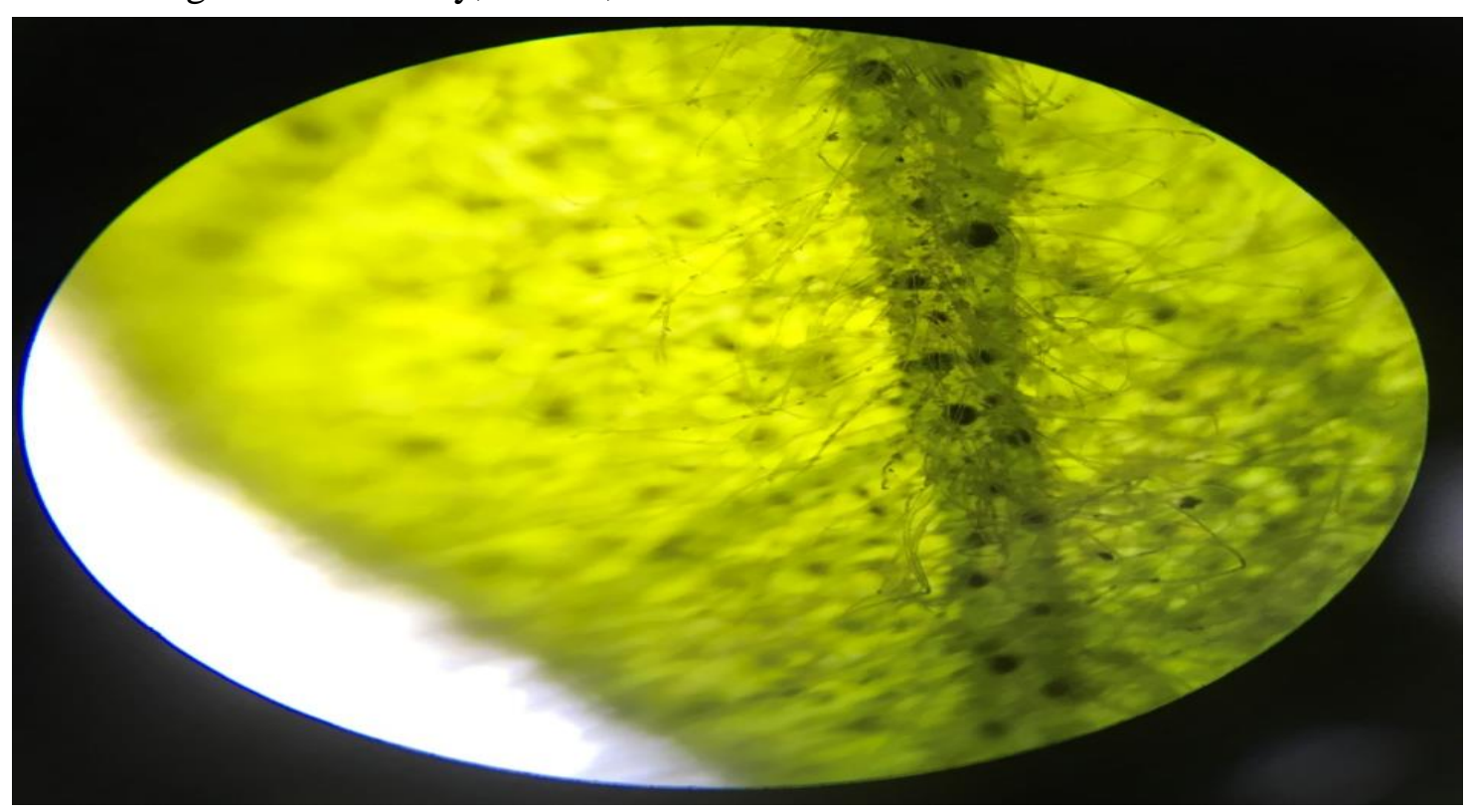

Figure 1. Trichome density and gossypol observation on mid rib on cotton leaf 


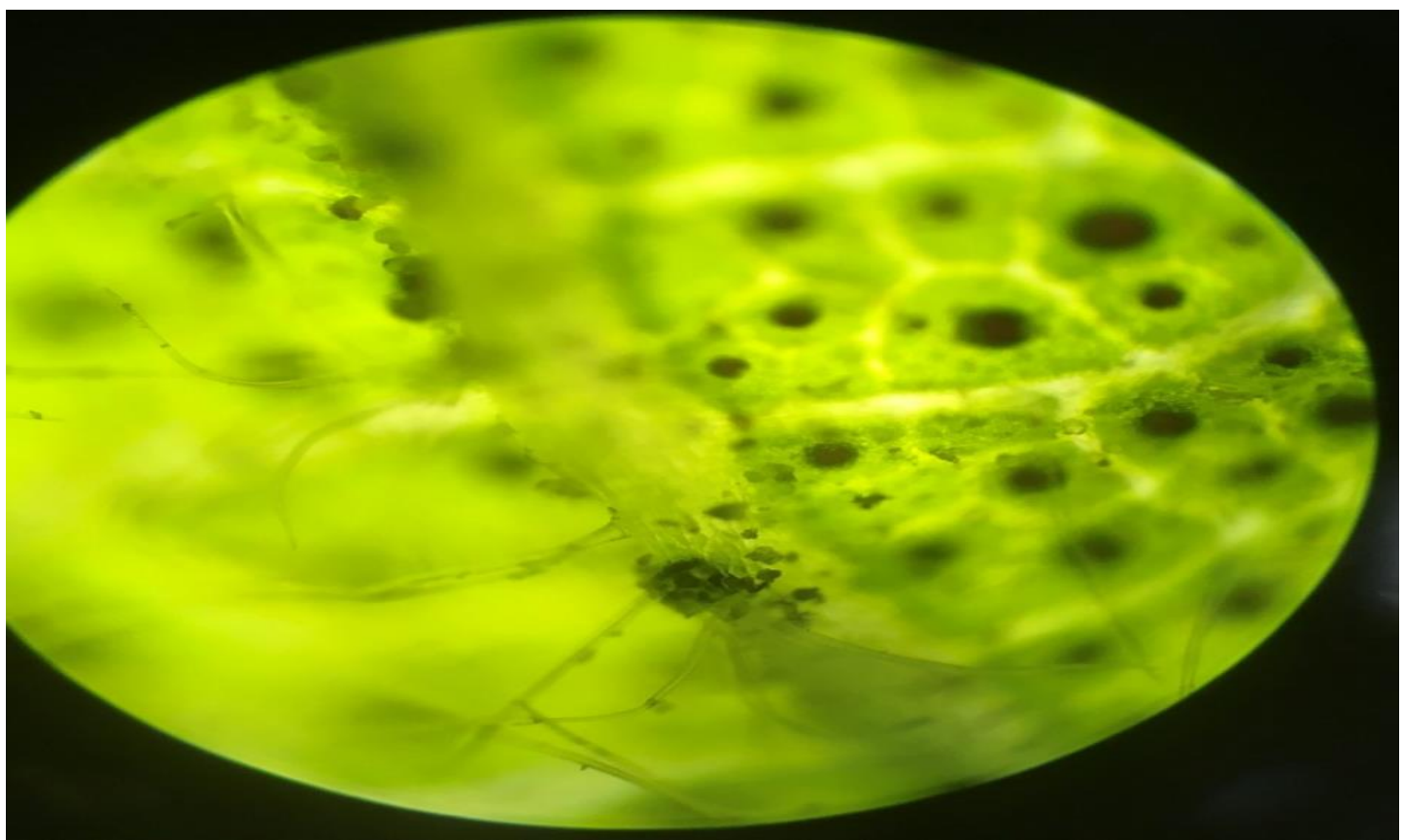

Figure 2. Gossypol and transparent trichomes observation on abaxial side of leaf

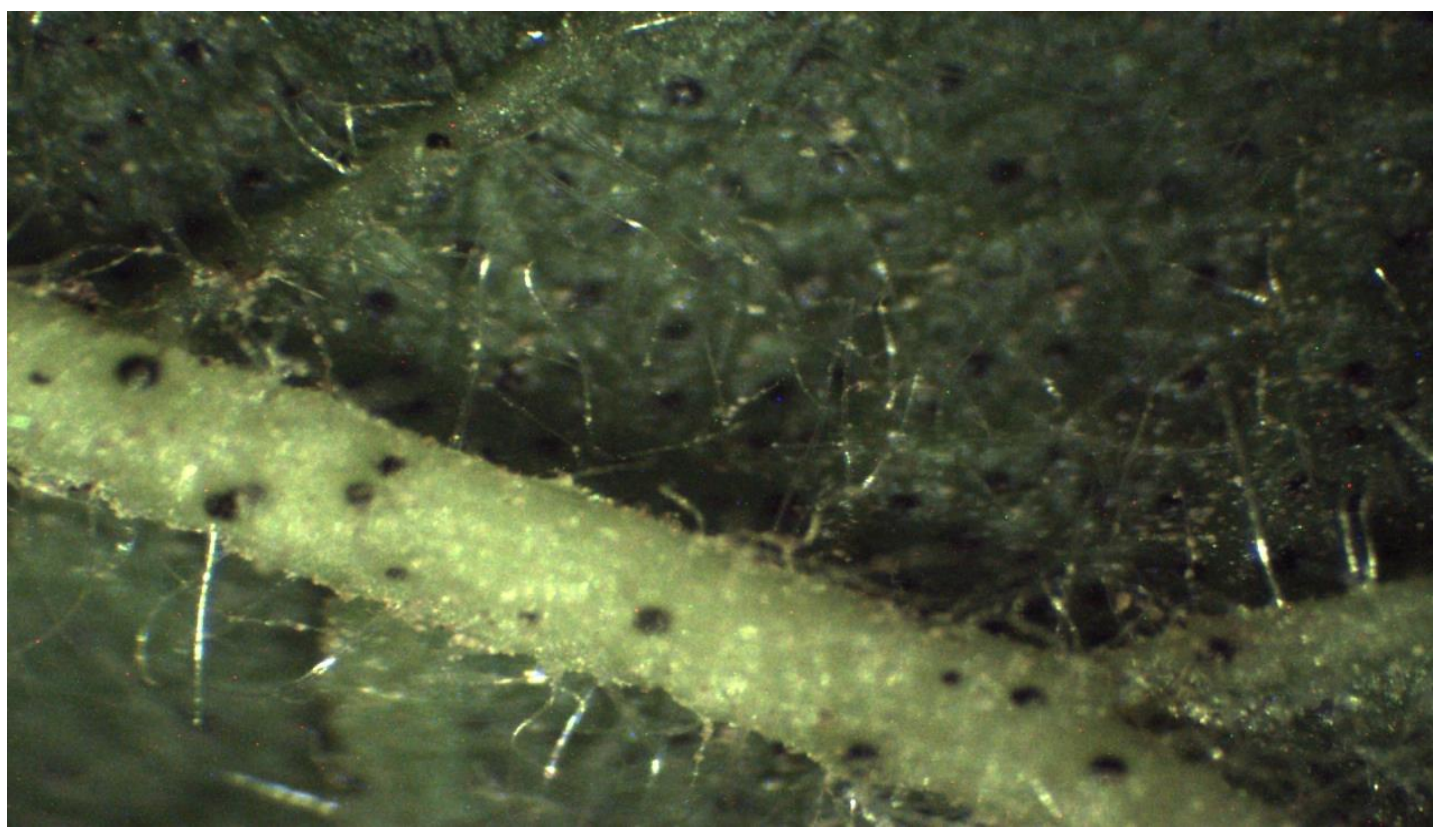

Figure 3. Trichome density on mid rib of the abaxial side of leaf of cotton plant

TRANSPARENT TESTA GLABRA (TTG) gene and transcriptional factors like MYB (myeloblastosis) transcriptional factor are important regarding trichomes production. Various phytohormones are also important in trichomes production. There are some negative regulators, which repress trichomes length and density like TTG1 and TTG2.

Trichomes are measured in scaling system. Those leaves with no trichomes are referred as smooth leaves or glabrous leaves. Trichome density is medium in Gossypium hirsutum leaf while leaves with maximum trichome density are called pilose [7]. This scaling system will help in classifying genotypes on the bases of hairiness. On the bases of this system, we can predict the resistance of genotype against sucking insects as it is reported that hairy varieties showed more resistance toward sucking insects [8]. 
Classification and morphology of trichomes

Trichomes are appendages, which may be glandular and non-glandular. In Arabidopsis, trichomes are glandless and only act as barrier to insects. While cotton have both glandular and non-glandular trichomes that can prevent insect attack in a better way. Glandular trichomes secrete alkaloids and other chemicals to protect plants from insect attack. These types of trichomes may vary among species while density and length of trichomes may change with factors like geography and their natural habitat [2].

Trichomes can also be classified on the bases of shape, as they can be straight, spiral, hooked, branched or un-branched. The structure of trichomes can range from unicellular to multi-cellular. Glandular trichomes secrete secondary metabolites like tannins, resins and some other sticky compounds, which repel insects and decrease the damage due to insect attack [2].

\section{Trichomes as a physical barrier against insects}

Trichomes are physical barriers against insect like leafhoppers and aphids as they secrete alkaloids like terpenes, which either kill the insects or retard their growth and population rate. Trichomes change the oviposition of insects in such a way that their reproduction rate reduces to minimum. In various species, there is a negative relationship between trichomes thickness and insect's oviposition reactions, and the larval hatchlings. Particular glandular trichomes may supplement the compound resistance of a plant by having organs, which produce terpenes, phenolic, alkaloids or different substance trichomes density [9]. Trichomes affect the chewing insects with small mouthparts as insect has to feed on trichomes first to expose epidermal portion of leave. Digestive system accumulates too many trichomes to digest which result ultimately in death of insects. Trichomes length, softness, hardness may vary in relation to insect attack. Trichomes secrete chemicals including chemicals, acyl sugars to kill or repel insects [10].

Plant trichomes also decrease chances of disease attack. As the chances of insect attack and invasion is less in plants with abundant trichomes. Trichomes secrete phenolic compounds which results in avoiding insect attack or at least minimizing insect attack.

\section{Genes responsible for trichomes regulation}

Work has been performed on model plant i.e. Arabidopsis regarding trichomes. MYB transcriptional factors has been found to be very important in inducing trichomes production. A. thaliana is model plant regarding trichomes production and its mechanism. Two genes are reported for trichomes initiation and spacing in leaves. These are GLABRA 1(GL1) and TRANSPARENT TESTA GLABRA 1(TTG1). These genes control hair formation in leaves in A. thaliana [11]. GLABROUS 2 and GLABROUS 3 are required for normal trichomes initiation and root hair development. ENHANCER OF GLABROUS, which codes for helical protein (BHL) is also required for trichomes initiation [12]. It is believed generally that same genes controlling trichomes in A. thaliana are responsible for trichomes initiation in cotton. GL1, MYB gene, BHL Protein complex are the main factors behinds trichomes production in cotton [3].

We can enhance trichomes production in our desired crop plants by disabling negative regulators. TCL1, TCL2, CPC are involved in suppressing trichomes production. If we disable their function, we can accelerate trichomes production. These genes suppresses GL1, which is directly involved in trichomes production hence trichomes production is suppressed [3].

\section{Role of phyto hormones in trichome regulation}

Jasmonic acid favours trichomes production while salicylic acid causes reduction in trichomes density. Jasmonic 
acid and gibberellic acid show synergistic effect in trichomes induction. When Jasmonic acid applied separately trichomes, induction is less as compared to collective application of jasmonic acid and gibberellic acid. When salicylic acid applied at the same time trichomes induction is not that high as in previous case [13].

Both artificial wound and herbivore attack result in increase in Jasmonic acid concentration, which then increases trichomes density. When only jasmonic acid is applied on Arabidopsis, there is an increase in trichomes density but this increase is comparatively lesser than when both gibberellic acid and jasmonic acid were applied [14]. Gibberellic acid is important hormone regarding plant growth and development.

\section{Trichomes response to herbivore attack}

Trichomes also defend plant in case of herbivore attack. When herbivore attacks on plant, Trichomes density and length increase in order to protect plant from further damage. Damages caused by herbivore are negatively correlated with trichomes production as trichomes density increases. But this is useful for plants as increased trichomes density protect plants from insect herbivore [2].

Both artificial wounding and herbivore attack resulted in increased in plant trichomes density and this behaviour is observed in both annual and perennial plants. The plants with herbivore attack produced trichomes with trichome density up to $500 \%$ while the one with no attack showed the trichomes density of about 20$40 \%$ only. Plant starts producing leaves in which trichomes density and length are more than previous leaves to assist plan in its survival against attack [15].

Insects who feed on epidermis through trichomes indicate increase mortality rate because of non-glandular trichomes. While glandular trichomes protect plants from lepidopteron and other insects by secreting certain chemical and alkaloids.

A strong relationship exists between trichome density and plant damage. It has been reported that potato varieties with smooth or glabrous leaves showed more infestation and damage caused by insects as compare to hairy varieties [8]. Plant varieties with more trichomes showed greater resistance to leafhopper attack [16].

\section{Role of trichome in a biotic stress}

Trichomes also play their role in biotic stress especially against drought. In low moisture content, trichomes help in water conservation in drought stress condition. Drought that results from excessive loss of water from leaf surface. Plant has to absorb water from soil through roots to fulfil plant requirement. The deleterious effects of drought stress can be minimized by enhancing trichomes density of leaves surface. Dense trichomes with the help of layer of air trapped in trichomes reduce the rate of water transpiration [17].

Plant species with more trichomes density also showed better performance in drought stress condition e.g. trichomes density and length is negligible in $G$. herkansii, $G$. Laxum, and abundant in G. annomalum. Hence, G. annomalum showed better performance in drought stress condition. Studies showed that plants with high trichomes showed greater water retention through unnecessary loss of water from plant and maintain the plant metabolism more effectively [18]. Cooling affect given by trichomes decrease water loss by 20 $25 \%$. This cooling affect also increase the carbon dioxide uptake.

Trichomes also confer against drought stress by minimize absorption of solar radiation and UV light and conserve plan moisture by minimizing loss of water from aerial parts of plant. Drought and increase in temperature effects plant processes like photosynthesis, evaporation and stomatal conductance. Photosynthesis efficiency is inversely affected by rise in temperature to ascertain limit.

\section{Trichome and fiber development}

A strong relationship has been found between fiber and trichome development. It has been observed that same promoter i.e., RDL1 gene is involved in fibre 
development and trichome development in cotton and Arabidopsis, respectively [19]. Cotton fibre is also considered as seed trichome and in fact the largest trichome in cotton. MYB transcriptional factors involved in fiber development have also very special role in trichome initiation and development.

\section{Measurement and rating system for trichome}

We can categorize plants in two ways either through scaling or through trichome number. In scaling system, there are three major classes of leaves. Leaves without trichomes are termed as glabrous. Leaves with medium density of trichomes are hirsute while leaves with maximum trichomes density are called pilose $[20,21]$ We can also count trichomes visually. Experiments showed that trichomes number may vary from 2 to 250 per $\mathrm{cm}^{2}$ [20]. A leaf with 5 trichomes per $\mathrm{cm}^{2}$ would be known as smooth leaf $[2,20]$.

\section{Conclusion}

Trichomes are important tool against sucking insect in cotton. Whitefly is the vector for $\mathrm{CLCuV}$ and trichomes can be used as a defence mechanism to prevent its attack by changing the oviposition of insects especially jassid. We can regulate trichomes development against abiotic stresses especially in drought by enhancing trichomes regulation either by exogenous application of phyto chemicals like Jasmonic acid or by over expression of genes (GL2) involved in trichome development. In Pakistan, local varieties do not possess very thick trichomes and it is also observed that it is hard to count trichomes in a specific area so we can classify leaves on the bases of morphological appearance. We suggest its time of the need that all the available cotton germplasm must be classify with respect to trichome density and length for further utilization inn breeding programmes.

\section{Authors' contributions}

Conceived and designed the experiments: M Shahzad, A Rasheed, W Nazeer \& Z Khan, Analysed the review paper: $M$
Tauseef, J Iqbal \& M Basheer, Contributed reagents/ materials/ analysis tools: $\mathrm{K}$ Hussain \& SI Hussain, Wrote the paper: M Shahzad, A Rasheed \& W Nazeer.

\section{References}

1. GOP G (2018). Pakistan Economic Survey 2017-18. Finance Division, Economic Advisor's Wing: Islamabad, Pakistan

2. Peter AJ, Shanower TG, Romeis J (1995). The role of plant trichomes in insect resistance: a selective review. Phytophaga, 7: 41-64.

3. Wang, S., J. Wang W., N. Yu., C. H. Li., B. Luo, Gou, J. Y. and X. Y. Chen. 2004. Control of plant trichome development by a cotton fiber MYB gene. The Plant Cell 16(9): 23232334.

4. Wu TC \& Kao WY (2009). The function of trichomes of an amphibious fern, Marsilea quadrifolia. American Fern J 99(4): 323-332.

5. Chen Y, Liu ZH, Feng L, Zheng Y, Li DD \& Li XB (2013). Genome-wide functional analysis of cotton (Gossypium hirsutum) in response to drought. PLoS One 8(11): e80879.

6. Tan J, Tu L, Deng F, Hu H, Nie Y \& Zhang X (2013). A genetic and metabolic analysis revealed that cotton fibre cell development was retarded by flavonoid naringenin. Plant Physiol 162(1): 86-95.

7. Nawab NN, Khan IA, Khan AA \& Amjad M (2011). Characterization and inheritance of cotton leaf pubescence. Pak J Bot 43(1): 649-658.

8. Gibson RW (1971). Glandular hairs providing resistance to aphids in certain wild potato species. Annals of Appl Biol 68(2): 113-119.

9. Levin DA (1973). The role of trichomes in plant defense. The quarterly review of biology 48(1): 315.

10. Handley R, Ekbom B \& Ågren J (2005). Variation in trichome density and resistance against a specialist insect herbivore in natural populations 
of Arabidopsis thaliana. Ecol Entomol 30(3): 284-292.

11. Oppenheimer DG, Herman PL, Sivakumaran S, Esch J \& Marks MD (1991). A myb gene required for leaf trichome differentiation in Arabidopsis is expressed in stipules. Cell 67(3): 483-493.

12. Zhang T, Hu Y, Jiang W, Fang L, Guan X, Chen J \& Hulse-Kemp AM (2015). Sequencing of allotetraploid cotton (Gossypiumhirsutum L. acc. TM-1) provides a resource for fiber improvement. Nature Biotechnol 33(5): 531.

13. Traw MB \& Bergelson J (2003). Interactive effects of jasmonic acid, salicylic acid, and gibberellin on induction of trichomes in Arabidopsis. Plant Physiol 133(3): 1367-1375.

14. Yoshida Y, Sano R, Wada T, Takabayashi J \& Okada K (2009). Jasmonic acid control of GLABRA3 links inducible defense and trichome patterning in Arabidopsis. Devel 136(6): $\quad$ 10391048.

15. Dalin P, Ågren J, Björkman C, Huttunen P \& Kärkkäinen K (2008). Leaf trichome formation and plant resistance to herbivory. In Induced plant resistance to herbivory (pp. 89105). Springer, Dordrecht.

16. Johnson HW \& Hollowell EA (1935). $\mathrm{Pu}$ - bescent and glabrous characters of soybeans as related to injury by the potato leafhopper. J Agr Res 51(2): 371-381

17. Xiao K, Mao X, Lin Y, Xu X, Zhu Y, Cai Q \& Zhang J (2017). Trichome, a functional diversity phenotype in plants. Mol Biol 6: 183.

18. Hanley ME, Lamont BB, Fairbanks MM \& Rafferty CM (2007). Plant structural traits and their role in antiherbivore defence. Persp in Plant Ecol, Evolution and System 8(4): 157-178.

19. Guan X, Pang M, Nah G, Shi X, Ye W, Stelly DM \& Chen ZJ (2014). miR828 and miR858 regulate homoeologous MYB2 gene functions in Arabidopsis trichome and cotton fibre development. Nature Comm 5(1): 1-14

20. Bourland FM, Hornbeck JM, Mc-Fall AB \& Calhoun SD (2003). A rating system for leaf pubescence of cotton. $J$ of Cotton Sci 7: 8-15.

21. Voigt D, Gorb E \& Gorb S (2007). Plant surface-bug interactions: Dicyphus errans stalking along trichomes. Arthropod-Plant

Interactions 1(4): 221-243. 